Pacific Journal of Mathematics

RINGS WITH INVOLUTION AND THE PRIME RADICAL 


\title{
RINGS WITH INVOLUTION AND THE PRIME RADICAL
}

\author{
W. E. Baxter and L. A. Casciotti
}

This paper first gives an equivalent characterization of the prime radical of $S$, the set of symmetric elements, in a ring $R$ which is 2 -torsion free and has an involution defined on it to that of Tsai by showing their equivalence using the results of Erickson and Montgomery.

With the ideas developed, the structure of Jordan ideals of $S$ under an algebraic condition similar to the topological condition previously investigated by Baxter is exhibited.

II. Two views of primeness and semiprimeness for Jordan ideals of $S$. Assuming that $R$ is a 2-torsion free ring with involution, we wish to characterize the prime radical of Tsai ([3] and [4]) differently. In so doing we offer a new definition of primeness and semiprimeness in the Jordan ring $S$ based on the (linear) Jordan multiplication $a \circ b=a b+b a$ (rather than the quadratic Jordan multiplication, $a U_{b}=b a b$, as investigated in [3]. We must emphasize however that the assumption of 2-torsion free is most important to this development. Henceforth $R$ is a 2-torsion free ring with involution, $a \rightarrow a^{*}$.

The definition given in [3] is due to Tsai [4], it is stated as follows.

\section{DEFINITION 1.}

(i) $Q$ is a $t$-prime ( $t$-semiprime) Jordan ideal of $S$ if whenever $A$ and $B$ are Jordan ideals of $S$ and $A U_{B}=\left\{\Sigma a U_{b} \mid a \in A, b \in B\right\} \subset Q$. Then either $A \subset Q$ or $B \subset Q\left(A U_{A} \subset Q\right.$ then $\left.A \subset Q\right)$.

(ii) $S$ is $t$-prime ( $t$-semiprime) if 0 is a $t$-prime (semiprime) Jordan ideal of $S$.

We wish to consider Jordan ideals of $S$ which are related to the quadratic multiplication

$$
\{u, s, v\}=s U_{u, v}=u s v+v s u .
$$

In this direction we make the following definition.

Definition 2. If $Q$ and $T$ are Jordan ideals of $S$ then

$$
Q \Lambda T=\{\Sigma\{q, s, t\} \mid q \in Q, s \in S, t \in T\}
$$

is the subgroup generated by $\{q, s, t\}$. 
It is easily shown that $Q \Lambda T$ is a Jordan ideal of $S$.

With this notation we introduce our definition of $j$-primeness ( $j$-semiprimeness).

\section{DEFINITION 3.}

(i) $Q$ is a $j$-prime ( $j$-semiprime) Jordan ideal of $S$ if whenever $A$ and $B$ are Jordan ideals of $S$ and $A A B \subset Q(A \Lambda A \subset Q)$ then either $A \subset Q$ or $B \subset Q(A \subset Q)$.

(ii) $S$ is $j$-prime ( $j$-semiprime) if, and only if, 0 is a $j$-prime ( $j$-semiprime) Jordan ideal of $S$.

One says that a subset $H$ is 2-divisible if whenever $2 x \in H$ then $x \in H$. The following follows very quickly.

LEMMA 4. If $Q$ is t-semiprime and 2-divisible Jordan ideal of $S$ then $Q$ is j-semiprime.

Suppose $A \Lambda A \subset Q$ for a Jordan ideal $A$ of $S$. Then, in particular, $\{a, b, a\} \in Q$ for all $a, b \in A$. That is, $2 a b a \in Q$. Since $Q$ is 2-divisible, $A U_{A} \subset Q$ and hence $A \subset Q$.

Following the same argument as Theorem 2, [3] one can prove

LEMMA 5. Let $I$ be a semiprime 2-divisible ideal of $R$ then $I \cap S$ is a j-semiprime Jordan ideal of $S$.

In particular, we observe that $\eta$, the prime radical, is a 2-divisible ideal of $R$ when $R$ is 2 -torsion free. This is stated as

LEMMA 6. Let $R$ be 2-torsion free with involution. Then the prime radical, $\eta$, is a two-divisible ideal of $R$. Hence, $\eta \cap S$ is j-semiprime.

The proof of the lemma follows by comparing $m$-sequences $\{x=$ $b_{0}, b_{i}=x c_{i} x$ for appropriately chosen $\left.c_{i}\right\}$ and $\left\{2 x=d_{0}, d_{i}=2^{(2 i)}\left(x c_{i} x\right)\right\}$ beginning at $x$ and $2 x$ respectively. If the latter vanishes $(R$ is 2 torsion free) so does the former. However, $x \notin \eta$ implies the existence of a non-vanishing $m$-sequence and $2 x \in \eta$ would contradict that fact.

Using the notion of $j$-semiprime, we introduce a new radical for $S$. We define 
Definition 7. $R_{j}(S)$ is the intersection of all the $j$-semiprime ideals of $S$.

We are now able to conclude

\section{THEOREM 8. $R_{j}(S)=\eta \cap S=\mathscr{L}(S)=\mathscr{P}(S)$.}

We know from [3], Lemma 1 that the latter two equalities hold. Furthermore, Lemma 6 tells us that $R_{j}(S) \subset \eta \cap S$. To show the inclusion the other way we make use of the argument of [3]. We modify it slightly to show that we can work with a more restrictive set; namely, $\mathscr{M}=\{M$ is a 2-divisible ideal of $R$, and $\left.M \cap S \subset R_{j}(S)\right\} . \quad 0 \in \mathscr{C}$ and thus $\mathscr{C} \neq \varnothing$. By Zorn's lemma, it has a maximal element, $M^{\prime}$. By construction, $M^{\prime}$ is 2-divisible. Furthermore, $M^{\prime}$ is semiprime. To see this, let $A$ be an ideal of $R, A^{2} \subset M^{\prime}, M^{\prime} \subset A$.

Thus, $(A \cap S) \Lambda(A \cap S) \subset A^{2} \cap S \subset M^{\prime} \cap S \subset R_{j}(S)$. As $R_{j}(S)$ is $j$ semiprime we have $A \cap S \subset R_{j}(S)$. If $A$ were 2-divisible we are done. If not, let $T=\left\{t \mid 2^{i} t \in A\right.$ for some positive integer $\left.i_{t}\right\}$. Then $T$ is a 2-divisible ideal of $R$ and $A \subset T$.

Now, $\left\{2^{t_{1}} t_{1}, s, 2^{t_{2}} t_{2}\right\} \in(A \cap S) \Lambda(A \cap S)$ for all $t_{1}, t_{2} \in T \cap S, s \in S$. Thus, $2^{t_{1}+t_{2}}\left\{t_{1}, s, t_{2}\right\} \in M^{\prime} \cap S$. However, $M^{\prime}$ is 2-divisible and so all the generators of $(T \cap S) \Lambda(T \cap S)$ are in the $j$-semiprime Jordan ideal $R_{j}(S)$. Therefore, $T \cap S \subset R_{j}(S)$ which contradicts the maximality of $M^{\prime}$. Hence, $M^{\prime}$ is a semiprime ideal of $R$. The following inclusions are obvious: $\eta \cap S \subset M^{\prime} \cap S \subset R_{j}(S)$. This completes the argument.

As a consequence of this theorem we have the following lemma whose proof is immediate.

Lemma 9. Let $I$ be $a^{*}$-ideal of $R$ such that $I \cap S=0$, then $I \subset K$ and $I^{3}=0$. In particular, if $\eta \cap S=0$ then $\eta \subset K$ and $\eta^{3}=0$.

We are now able to conclude that in 2-torsion free rings with involution these definitions of primeness (and semiprimeness) are equivalent.

THEOREM 10. Let $R$ be 2-torsion free with involution. Then $S$ is t-prime if, and only if, $S$ is j-prime.

The easier direction appears to be that $t$-prime implies $j$-prime. Thus, assume $S$ is $t$-prime and let $A A B=0$ for Jordan ideals $A$ and $B$ of $S$. Now for $a, b \in A \cap B$ we have $\{a, b, a\}=0$. Thus $(A \cap$ $B) U_{A \cap B}=0$. That is, $A \cap B=0$. Therefore $2\{a, b, a\}=0$ for all 
$a \in A, b \in B$. That is, $B U_{A}=0$. Hence, by hypothesis, $A=0$ or $B=0$. This is the desired conclusion.

To see the other direction, assume $S$ is $j$-prime (then, of course, $S$ is $j$-semiprime). Thus by Theorem $8, \eta \cap S=0$. That is, $S$ is $t$ semiprime. Let $A$ and $B$ be Jordan ideals of $S$ such that $A U_{B}=0$. Then $(A \cap B) U_{(A \cap B)}=0$ or $A \cap B=0$. Therefore, for all $a \in A, b \in B, \alpha \circ b=0$.

Thus, $a \circ(b \circ s)=0=(a \circ s) \circ b$ for all $a \in A, b \in B, s \in S$.

Expanding, and adding, we have $\{a, s, b\}=0$ for all $a \in A, s \in S$, $b \in B$. That is, $A A B=0$. Since $S$ is $j$-prime we have the desired conclusion.

We next investigate semiprimeness of $S$ and show that each definition implies the other.

THEOREM 11. Let $R$ be 2-torsion free with involution. Then $S$ is t-semiprime if, and only if, $S$ is $j$-semiprime.

If $S$ is $t$-semiprime and $A \Lambda A=0$ for a Jordan ideal of $S$, we can immediately conclude by appropriate choices that $A U_{A}=0$. Hence, $A=0$. That is, $S$ is $j$-semiprime.

The argument in the other direction assumes that $S$ is $j$-semiprime, that is, 0 is $j$-semiprime ideal and hence $R_{j}(S)=0=\eta \cap S$. Let $A$ be a Jordan ideal of $S$ with the property that $A U_{A}=0$. Recalling the definitions and conclusions of [1], we have $B=\{b \mid b a+$ $\left.a^{*} b^{*} \in A\right\}$ is a right ideal of $R, S B \subset B$, and hence $B \cap S$ is a Jordan ideal of $S$. Thus, $B \cap A$ is a Jordan ideal of $S$ and for all $b$, $c \in B \cap A, a \in R$ we have both $c\left(b a+a^{*} b\right) c=0$ and $b c b=0$. Therefore, $b c\left(b a+a^{*} b\right) c=0$, or $b c R b c=0$. That is, $b c \in \eta$ and so $b \circ c \in \eta \cap S=0$.

Since $R$ is 2-torsion free we are able to conclude that $b \circ c=b^{2}=0$ for all $b, c \in B \cap A$. Therefore, $b \circ(b \circ s)=2 b s b=0$ for all $b \in B \cap A$, $s \in S$.

That is, $(B \cap A) U_{(B \cap A)}=0$ and so the conclusion, $B \cap A=0$.

However, $4(A \circ A) \subset B \cap A=0$, and so $A \circ A=0$, Hence, as above, we conclude that $A=0$. That is, we have shown that $S$ is $t$-semiprime when $S$ is $j$-semiprime.

III. An annihilator condition on $S$. In this section we consider the results of [2] after removing the topological conditions on the ring, $R$, and the requirements that $2 R=R, R^{2}=R$, and $R$ is semi-prime.

We continue the hypothesis that $R$ is a 2-torsion free ring with involution and $S$ is t-semiprime. Therefore, the prime radical, $\eta$, is a two-divisible ideal of $R$, contained in $K$. That is, $\eta \cap S=0$ and $\eta^{3}=0$.

Hence, in $\bar{R}=R / \eta$ we have the property that $2 S_{\bar{R}} \subset \bar{S}_{R}$. One 
uses this remark to conclude that

LEMMA 12. Let $R$ be a 2-torsion free ring with involution. Let $S$ be t-semi-prime. Let $A$ be a Jordan ideal of $S$ with the property that $a^{n}=0$ for all $a \in A, n$ a fixed positive integer. Then $A=0$.

As in [1] and [2] we continue the notation

$$
(a, b)_{J}=a b+b^{*} a^{*} \text { for all }
$$

$a, b \in R$.

In $R / \eta$ we can assume that an element of $\overline{B_{A}}$ comes from an element of $B_{A}$. Indeed, notice that

$$
\eta \subset B_{A}=\left\{b \mid(b, a)_{J} \subset A\right\} .
$$

This is a consequence of $(n, a)_{J}=0$ for all $n \in \eta, a \in R$.

Letting $\mathscr{L}\left(\bar{B}_{A}\right)$ denote the left-annihilator of $\bar{B}_{A}$ in $R / \eta$, we can conclude that $\mathscr{L}_{N}(B)=\{t \mid t b \in \eta$ for all $b \in B\}$ is a set which inherits the properties of $\mathscr{L}(B)$ of [2]. These are collected as the following theorem.

THEOREM 13. Let $R$ be a 2-torsion free ring with involution. Let $S$ be t-semiprime. Let $A$ be a Jordan ideal of $S$ and let $B=B_{A}$ and $\mathscr{L}_{N}(B)$ be as defined then:

(i) $\mathscr{L}_{N}(B)$ is a two sided self adjoint ideal of $R$.

(ii) $\mathscr{L}_{N}(B) \cap B=\eta$

(iii) If $\mathscr{L}_{N}(V)=\{t \in R \mid t v \in \eta$ for all $v$ in the Jordan ideal $V$ of $S\}$, then $\mathscr{L}_{N}(V)=\mathscr{L}_{N}(B \cap S)=\mathscr{L}_{N}(B \cap V)=\mathscr{L}_{N}(B)$

(iv) If $V$ is a Jordan ideal of $S$ and $\mathscr{A}_{J}(V)=\{t \in S \mid t \circ v=0$ for all $v \in V\}$; the Jordan annihilator of $V$ in $S$, then $\mathscr{L}_{N}(V) \cap S=\mathscr{A}_{J}(V)$.

(v) If $W=\left(\mathscr{L}_{N}(B) \cap S\right) \oplus(B \cap S)$ then $\mathscr{A}_{J}(W)=0$.

(vi) If $Y=\left(\mathscr{L}_{N}(B) \cap S\right) \oplus(A \cap S)$ then $\mathscr{A}_{J}(Y)=0$.

We now impose an algebraic annihilator condition on $S$ which is related to the annihilator condition of [2]; namely.

Definition 14. $A$ ring $R$ is said to satisfy condition $\mathscr{A}$ if, and only if,

(i) $R$ is 2-torsion free with involution,

(ii) $S$ is $t$-semiprime, and

(iii) whenever $V$, a Jordan ideal of $S$, is such that $\mathscr{A}_{J}(V)=0$ then $V=S$. 
Using this concept we have

LEMma 15. Let $R$ satisfy condition $\mathscr{A}$ then:

(i) If $L$ is a Jordan ideal of $S$ such that for all $t \in \mathscr{A}_{J}(L)$, $t^{n} \in L$ ( $n$ a fixed positive integer) then $L=S$.

(ii) The norms generate $S$, the traces generate $S, R^{n} \cap S=S$ for all positive integers $n$.

(iii) $S=\left(B_{V} \cap S\right) \oplus\left(\mathscr{L}_{N}(B) \cap S\right)=V \oplus \mathscr{L}_{N}(V)$ for all Jordan ideals $V$ of $S$.

Remarks (ii) and (iii) follow immediately from (i). Hence, we prove (i). Suppose $t^{n} \in \mathcal{L}$ for all $t \in \mathscr{A}_{J}(L)$. Then, $t^{n} \circ t=0$ for all $t \in \mathscr{A}_{J}(L)$. That is, $t^{n+1}=0$ for all $t \in \mathscr{A}_{J}(L)$. By Lemma 12, $\mathscr{A}_{J}(L)=0$ or $L=S$.

This annihilator condition is sufficient to guarantee that $B_{V}$ is a two-sided ideal for any Jordan ideal, $V \subset S$. This remark forms a part of the following theorem.

THEOREM 16. Let $R$ satisfy condition $\mathscr{A}$. Then $B_{V} \cap S$ and $\mathscr{L}_{N}(B) \cap S$ are strongly semiprime Jordan ideals of $S, V=B_{V} \cap S$, and $B_{V}$ is a two-sided, self-adjoint ideal of $R$.

Letting $B_{V}=B$, and $\mathscr{L}_{N}(B)=L$ one observes that for all $b \in B \cap S, s \in S, t \in L \cap S$,

$$
\{b, s, t\}=0 \text {. }
$$

Since, $S=(B \cap S) \oplus(L \cap S)$ we have for each $a \in S, a=b+t$, for some $b \in B \cap S, t \in L \cap S$. Now if $r U_{a}=a r a \in B \cap S$ for all $r \in S$, we must have (using the above observation)

$$
r U_{t} \in(B \cap L) \cap S=0 \text {. }
$$

That is, $t=0$ and hence $a=b \in B$.

Now, if $u=b+t$ for some $b \in B \cap S, t \in L \cap S$ then

$$
(u \circ s)-(b \circ s)=(t \circ s) \in V \cap L
$$

for all $s \in S$. Thus, $t \in \mathscr{A}_{J}(S)$ or $t=0$. Hence, $V \subset B$. On the other hand, arguing from $S=V \oplus(L \cap S)$ we conclude $B \cap S \subset V$.

Furthermore, given $b \in B, a \in R$ then $(b, a)_{J} \in B \cap S=V$. However, $B$ is a right ideal. Thus, $R b^{*} \in B$ for all $b \in B$. Now consider $a b$ for arbitrary $a \in R, b \in B$. Then, for all $c \in R$ consider

$$
((a b), c)_{J} \text {. }
$$

We observe, $((a b), c)_{J}=\left(\left(c^{*} b^{*}\right), a^{*}\right)_{J} \in V$. Hence, $a b \in B$. That is, $B$ is a two-sided ideal of $R$. 
Now, for any $b \in B, b+b^{*}=c+t$ for appropriate $c \in B \cap S$, $t \in L \cap S$. Therefore $t U_{s}=(b-c) U_{s}+b^{*} U_{s}$. Since $R b^{*} \in B$ we have the right side of this expression is in $B$. Therefore, $t U_{s}=0$ for all $s \in S$. It follows that $V=\left\{t \in S \mid t U_{s}=0\right\}$ is a Jordan ideal and $V U_{V}=0$. Hence $V=0$ as $S$ is $t$-semiprime. Thus, $t=0$, or $b^{*} \in B$. That is, $B$ is self-adjoint.

This theorem has the interesting consequence which was observed in [2].

THeOREM 17. Let $R$ satisfy condition $\mathscr{A}$. Let $V$ be the additive subgroup generated by the elements $\left\{u \alpha-a^{*} u \mid u \in W\right.$, a nonzero Jordan ideal of $S$, and $a \in R$. Then $W+V$ contains a nonzero 2-sided ideal of $R$. Furthermore, if $W$ is minimal, $2 R=R$, or $2 W=W$ this last containment is an equality.

The proof of the first fact is to observe that since $B \cap S=W$ we have $R(2 u) R \subset W+V$ for all $u \in W$. Since $S$ is $t$-semiprime, we can conclude that $R(2 u) R \neq 0$ for some $u \in W$ and hence the desired result. If $W$ is minimal, $2 W=W$ and hence the latter remarks follow quickly.

THEOREM 18. Let $R$ be a semiprime ring with condition $\mathscr{A}$. Let $W$ be a Jordan ideal of $S$. Then either $W \subset \zeta^{a}$ (the annihilator of the socle) or $W$ contains an idempotent.

The proof is the same as in [2]. Knowing $B \cap S=W$, we can conclude that whenever a symmetric idempotent is in the set on the left then it is in the set on the right. This observation replaces the need for the assumption $2 R=R$.

\section{REFERENCES}

1. W. E. Baxter, On rings with proper involution, Pacific J. Math., 27 (1968), 1-12.

2. - Topological rings with property $Y$, Pacific J. Math., 30 (1969), 563-571.

3. T. S. Erickson and S. Montgomery, The prime radical in special Jordan rings, Trans. Amer. Math. Soc., 156 (1971), 155-164.

4. C. Tsai, The prime radical in a Jordan ring, Proc. Amer. Math. Soc., 19 (1968), 1171-1175.

Received June 1, 1976 and in revised form October 13, 1976. The first author's work were supported in part by NSF Grant No. GP-9611. Portions of the second author's work form a portion of a PH. D. dissertation submitted to the Faculty of the University of Delaware.

University of Delaware

AND

Wilmington CoLlege 



\section{PACIFIC JOURNAL OF MATHEMATICS}

EDITORS

RICHARD ARENS (Managing Editor)

University of California

Los Angeles, California 90024

R. A. Beaumont

University of Washington

Seattle, Washington 98105
J. DUGUNDJI

Department of Mathematics

University of Southern Californı

Los Angeles, California 90007

D. Gilbarg and J. Milgram

Stanford University

Stanford, California 94305

\section{ASSOCIATE EDITORS}

E. F. BECKENBACH

B. H. NeumanN

F. WOLF

K. YoSHIDA

\section{SUPPORTING INSTITUTIONS}

UNIVERSITY OF BRITISH COLUMBIA

CALIFORNIA INSTITUTE OF TECHNOLOGY

UNIVERSITY OF CALIFORNIA

MONTANA STATE UNIVERSITY

UNIVERSITY OF NEVADA

NEW MEXICO STATE UNIVERSITY

OREGON STATE UNIVERSITY

UNIVERSITY OF OREGON

OSAKA UNIVERSITY
UNIVERSITY OF SOUTHERN CALIFORNIA

STANFORD UNIVERSITY

UNIVERSITY OF TOKYO

UNIVERSITY OF UTAH

WASHINGTON STATE UNIVERSITY

UNIVERSITY OF WASHINGTON

AMERICAN MATHEMATICAL SOCIETY

NAVAL WEAPONS CENTER 


\section{Pacific Journal of Mathematics}

Vol. 69 , No. 1

May, 1977

V. V. Anh and P. D. Tuan, On starlikeness and convexity of certain analytic

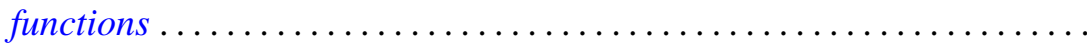

Willard Ellis Baxter and L. A. Casciotti, Rings with involution and the prime

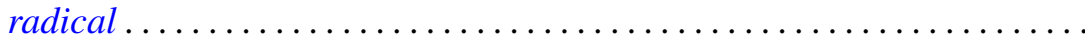

Manuel Phillip Berriozabal, Hon-Fei Lai and Dix Hayes Pettey,

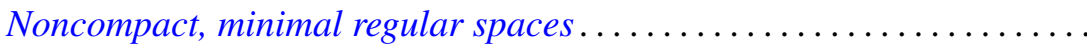

Sun Man Chang, Measures with continuous image law ................

John Benjamin Friedlander, Certain hypotheses concerning

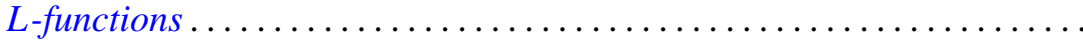

Moshe Goldberg and Ernst Gabor Straus, On characterizations and

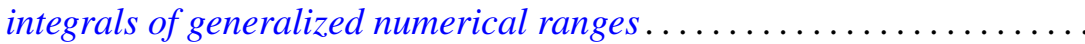

Pierre A. Grillet, On subdirectly irreducible commutative semigroups ...... 55

Robert E. Hartwig and Jiang Luh, On finite regular rings ..............

Roger Hugh Hunter, Fred Richman and Elbert A. Walker, Finite direct sums of cyclic valuated p-groups ........................... 97

Atsushi Inoue, On a class of unbounded operator algebras. III ......... 105

Wells Johnson and Kevin J. Mitchell, Symmetries for sums of the Legendre

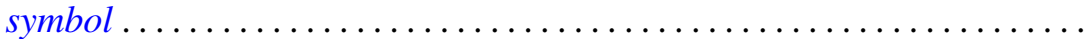

Jimmie Don Lawson, John Robie Liukkonen and Michael William Mislove,

Measure algebras of semilattices with finite breadth

Glenn Richard Luecke, A note on spectral continuity and on spectral properties of essentially $G_{1}$ operators ...............

Takahiko Nakazi, Invariant subspaces of weak-* Dirichlet algebras . .

James William Pendergrass, Calculations of the Schur group ...

Carl Pomerance, On composite $n$ for which $\varphi(n) \mid n-1$. II. . .

Marc Aristide Rieffel and Alfons Van Daele, A bounded operator approach to Tomita-Takesaki theory........................

Daniel Byron Shapiro, Spaces of similarities. IV. $(s, t)$-families ...

Leon M. Simon, Equations of mean curvature type in 2 independent variables.

Joseph Nicholas Simone, Metric components of continuous images of ordered compacta ............................

William Charles Waterhouse, Pairs of symmetric bilinear forms in

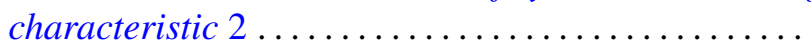

